comorbidities, haemoglobin levels, vitamin $\mathrm{B}_{12}$, folate, ferritin and albumin levels. A positive result was a rise in hydrogen of at least $20 \mathrm{ppm}$, or methane of $12 \mathrm{ppm}$, over the baseline for each gas.

Results 447 patients were identified (120 male, median age 56 years, range $17-90)$. Overall $84 / 447$ (18.8\%) of tests were positive. The patient characteristics associated with a positive result were concurrent use of proton pump inhibitor (PPI) $(p=0.0005)$, previous partial gastrectomy $(p<0.0001)$, previous right hemicolectomy $(p=0.0004)$, and age over 75 tears $p<0.0001$. The laboratory investigations predictive of a positive result were low vitamin $B_{12}$ $(\mathrm{p}=0.02)$ and low albumin $<30 \mathrm{~g} / \mathrm{dl}(\mathrm{p}=0.03)$.

Conclusion This is the largest single centre study of factors predictive of SIBO as defined by positive GHBT. Use of proton pump inhibitor, partial gastrectomy, right hemicolectomy, age over 75 years, low vitamin $\mathrm{B}_{12}$ and low albumin were predictive of SIBO.

Competing interests None declared.

\section{PWE-116 CAN THE NEW ESPGHAN DIAGNOSTIC GUIDELINES FOR COELIAC DISEASE BE APPLIED TO ADULTS?}

doi:10.1136/gutjnl-2012-302514d.116

L L Wong, ${ }^{*}$ R Sood, G Robins. Department of Gastroenterology, York District Hospital, York, UK

Introduction In adults, duodenal biopsy is mandatory for diagnosis of coeliac disease. This is usually preceded by serological tests for coeliac specific antibodies-anti-tissue transglutaminase (tTG) and endomysial antibodies (EMA). However, the recent guidelines produced by the European Society for Paediatric Gastroenterology, Hepatology and Nutrition (ESPGHAN) ${ }^{1}$ in 2010 recommend that histological assessment (duodenal biopsy) might be omitted in cases of anti-tTG more than 10 times the upper limit of normal which have been verified by endomysial positivity, a typical history for coeliac disease and HLA DQ2 or DQ8 positivity. Our aim was to analyse our adult population of patients with possible diagnosis of coeliac disease to ascertain the proportion of patients with anti-tTG levels more than 10 times the upper limit of normal.

Methods Retrospective collection of data were obtained from the electronic clinical patient database from 2008 to 2011 at York Hospital. Inclusion criteria were all patients with positive anti-tTG and available duodenal histology results while on normal diet. York Hospital uses the Orgentec Anti-tTG IgA kit.

Results $113(70 \%)$ of the 161 patients were female. The median age was 49 years (Range 16-89 years). 52 patients (32\%) had anti-tTG levels greater than 10 times the upper limit of normal (ie, with a value $\geq 100 \mathrm{u} / \mathrm{ml}$ ). All 52 had positive EMA. 51/52 had typical symptoms (chronic diarrhoea, weight loss, fatigue, anaemia) while one patient had leg weakness and a positive family history for coeliac disease. All 52 patients had biopsies consistent with coeliac disease. One patient had HLA DQ2/DQ8 status checked, which was positive. The test was done due to equivocal IgA levels, weakly positive anti-tTG and Marsh I findings.

Conclusion In our local adult population of patients with known positive anti-tTG and duodenal histology, about a third of the patients had anti-tTG levels greater than 10 times the upper limit of normal. This group had biopsies consistent with coeliac disease. Therefore, the ESPGHAN diagnostic guideline recommending the omission of duodenal biopsies in patients with anti-tTG levels greater than 10 times the upper limit of normal may be applicable for a significant proportion of the adult population (other similar studies $^{2} 3$ have found rates of $45-58 \%$ ).

Competing interests None declared.

\section{REFERENCES}

1. ESPGHAN Guidelines Diagnosis Coeliac Disease Children/Adolescents. 2010. http:// www.celiac-disease-consortium.nl/attachments/080_Mearin\%20ESPGHAN\%20CD\% 20WG\%20voor\%20CDC\%20november\%202010.pdf

2. Mubarak A, Wolters VM, Gerritsen SA, et al. A biopsy is not always necessary to diagnose celiac disease. J Pediatr Gastroenterol Nutr 2011;52:554-7.

3. Hill PG, Holmes GK. Coeliac disease: a biopsy is not always necessary for diagnosis Aliment Pharmaco Ther 2008:27:572-7.

\section{PWE-117 RESPONSES TO DIETARY INTERVENTION GUIDED BY FOLLOW-UP DUODENAL BIOPSY IN COELIAC DISEASE}

doi:10.1136/gutjnl-2012-302514d.117

${ }^{1} \mathrm{~L}$ Sharkey, ${ }^{*} \mathrm{G}$ Corbett, ${ }^{2} \mathrm{E}$ Currie, ${ }^{2} \mathrm{~J}$ Lee, ${ }^{3} \mathrm{~N}$ Sweeney, ${ }^{1} \mathrm{~J}$ Woodward. ${ }^{1}$ Department of Gastroenterology and Clinical Nutrition, Addenbrooke's Hospital, Cambridge, UK; ${ }^{2}$ Department of Nutrition and Dietetics, Addenbrooke's Hospital, Cambridge, UK, ${ }^{3}$ University of Adelaide, Adelaide, Australia

Introduction British Society of Gastroenterology guidance on repeating the intestinal biopsy in coeliac patients on diet is ambiguous and suggests that dietary advice is the same irrespective of the follow-up biopsy results. Addenbrooke's Coeliac Disease Clinic has a policy of routine re-biopsy at 9-12 months after commencing gluten withdrawal. The following graded dietary interventions are introduced if villous atrophy is present on repeat biopsy-interview and dietary history; food diary; withdrawal of oats; withdrawal of "Codex" products, barley malt extract, ("supersensitive diet"—SSD); liquid diet. Response is assessed by re-biopsy as we have shown serology to be unreliable in this setting.

Methods Information was retrieved from the Addenbrooke's Adult Coeliac Disease database of over 600 patients with coeliac disease (30\% male, $70 \%$ female, average age at diagnosis 48 yrs and $44 \mathrm{yrs}$ respectively). 170 patients with persisting villous atrophy on followup biopsy were identified. Dietetic interventions and outcomes based on subsequent biopsy results were reported.

Results Of 170 patients with persisting villous atrophy, 84 did not undergo re-biopsy after dietitian intervention and were therefore excluded from further analysis. In 57 patients interview or food diary analysis revealed a likely source of gluten ingestion as the cause of persistent villous atrophy and advice was given to eliminate the likely source (Dietary Advice-DA). In 29 patients, no potential gluten source was identified and a "supersensitive diet" was recommended (SSD). Further biopsy revealed complete normalisation of the duodenal mucosa (Marsh 0 ) in 14 (24.5\%) of the DA group and $8(27.5 \%)$ of the SSD group. Normal or minor changes (Marsh 0, 1, or 2) were seen in 31 (54\%) of the DA group and $18(62 \%)$ of the SSD group. Intensive dietary intervention revealed two additional patients who concealed deliberate gluten ingestion. 11 patients who remained persistently seronegative, had no identifiable source of gluten and showed no response to SSD were deemed to be histologically refractory and assigned to careful clinical follow-up.

Conclusion Contrary to current BSG guidance, dietary advice is not irrespective of the outcome of the follow-up biopsy on gluten free diet. Dietitian intervention is effective in over half of the patients who showed persistent villous atrophy despite following a gluten free diet. This strategy also identifies patients who are histologically refractory and at high risk of subsequent complications. These results strongly support a policy of assessment by follow-up biopsy and appropriate specialist dietitian intervention in the management of coeliac disease.

Competing interests None declared. 